\title{
Homozygous mutation in SLO3 leads to severe asthenoteratozoospermia due to acrosome hypoplasia and mitochondrial sheath malformations
}

Mingrong Lv ${ }^{1,2,3+}$ (0), Chunyu Liu ${ }^{4,5,6+}$, Chunjie Ma ${ }^{7 \dagger}$, Hui Yu ${ }^{8 \dagger}$, Zhongmei Shao ${ }^{8 \dagger}$, Yang Gao ${ }^{2,3,9}$, Yiyuan Liu ${ }^{1,2,3}$, Huan Wu $\mathbf{W}^{1,2,3}$, Dongdong Tang ${ }^{1,2,3}$, Qing Tan ${ }^{1,10}$, Junqiang Zhang ${ }^{2,3,9}$, Kuokuo Li ${ }^{1,2,9}$, Chuan Xu ${ }^{1,2,3}$, Hao Geng 1,2,3, Jingjing Zhang ${ }^{1,2,3}$, Hang $\mathrm{Li}^{1,10}$, Xiaohong Mao ${ }^{1,10}$, Lei Ge $\mathrm{G}^{1,10}$, Feifei Fu ${ }^{1,10}$, Kaixin Zhong ${ }^{7}$, Yuping Xu ${ }^{1,2,3}$, Fangbiao Tao ${ }^{2,3}$, Ping Zhou ${ }^{1,2,3}$, Zhaolian Wei ${ }^{1,2,3}$, Xiaojin He ${ }^{1,2,3,10^{*}}$, Feng Zhang ${ }^{4,5,6^{*}}$ and Yunxia Cao ${ }^{1,2,3^{*}}$

\begin{abstract}
Background: Potassium channels are important for the structure and function of the spermatozoa. As a potassium transporter, the mSlo3 is essential for male fertility as Slo3 knockout male mice were infertile with the series of functional defects in sperm cells. However, no pathogenic variant has been detected in human SLO3 to date. Here we reported a human case with homozygous $\mathrm{SLO} 3$ mutation. The function of $\mathrm{SLO} 3$ in human sperm and the corresponding assisted reproductive strategy are also investigated.

Methods: We performed whole-exome sequencing analysis from a large cohort of 105 patients with asthenoteratozoospermia. The effects of the variant were investigated by quantitative RT-PCR, western blotting, and immunofluorescence assays using the patient spermatozoa. Sperm morphological and ultrastructural studies were conducted using haematoxylin and eosin staining, scanning and transmission electron microscopy.
\end{abstract}

Results: We identified a homozygous missense variant (c.1237A > T: p.lle413Phe) in the sperm-specific SLO3 in one Chinese patient with male infertility. This $S L O 3$ variant was rare in human control populations and predicted to be deleterious by multiple bioinformatic tools. Sperm from the individual harbouring the homozygous $\mathrm{SLO} 3$ variant exhibited severe morphological abnormalities, such as acrosome hypoplasia, disruption of the mitochondrial sheath, coiled tails, and motility defects. The levels of SLO3 mRNA and protein in spermatozoa from the affected individual were reduced. Furthermore, the acrosome reaction, mitochondrial membrane potential, and membrane potential during capacitation were also afflicted. The levels of acrosome marker glycoproteins and PLC 1 as well as the mitochondrial

\footnotetext{
*Correspondence: hxj0117@126.com; zhangfeng@fudan.edu.cn; caoyunxia6@126.com

${ }^{\dagger}$ Mingrong Lv, Chunyu Liu, Chunjie Ma, Hui Yu and Zhongmei Shao contributed equally to this work.

1 Reproductive Medicine Center, Department of Obstetrics

and Gynecology, the First Affiliated Hospital of Anhui Medical University,

Hefei 230022, Anhui, China

${ }^{4}$ Obstetrics and Gynecology Hospital, State Key Laboratory of Genetic

Engineering at School of Life Sciences, Institute of Reproduction and Development, Fudan University, Shanghai 200011, China

Full list of author information is available at the end of the article
}

(c) The Author(s) 2021. Open Access This article is licensed under a Creative Commons Attribution 4.0 International License, which permits use, sharing, adaptation, distribution and reproduction in any medium or format, as long as you give appropriate credit to the original author(s) and the source, provide a link to the Creative Commons licence, and indicate if changes were made. The images or other third party material in this article are included in the article's Creative Commons licence, unless indicated otherwise in a credit line to the material. If material is not included in the article's Creative Commons licence and your intended use is not permitted by statutory regulation or exceeds the permitted use, you will need to obtain permission directly from the copyright holder. To view a copy of this licence, visit http://creativecommons.org/licenses/by/4.0/. The Creative Commons Public Domain Dedication waiver (http://creativeco mmons.org/publicdomain/zero/1.0/) applies to the data made available in this article, unless otherwise stated in a credit line to the data. 
sheath protein HSP60 and SLO3 auxiliary subunit LRRC52, were significantly reduced in the spermatozoa from the affected individual. The affected man was sterile due to acrosome and mitochondrial dysfunction; however, intracytoplasmic sperm injection successfully rescued this infertile condition.

Conclusions: SLO3 deficiency seriously impact acrosome formation, mitochondrial sheath assembly, and the function of $\mathrm{K}^{+}$channels. Our findings provided clinical implications for the genetic and reproductive counselling of affected families.

Keywords: Infertility, Asthenoteratozoospermia, SLO3, Acrosome hypoplasia, Mitochondrial sheath malformation

\section{Introduction}

Infertility is a significant global health issue affecting approximately $10-15 \%$ of couples worldwide, with the male factor being a component in at least $50 \%$ of couples. Studies have shown that many genetic and epigenetic factors play a role in male infertility, but many cases occur due to unknown reasons [1].

Many factors have been found to influence sperm motility and morphology, and genetic defects in the sperm result in the development of asthenoteratozoospermia [2]. Potassium channels are important factors in the structure and function of the sperm, and their defects can cause dysfunction in the physiology and function of sperm. As a potassium transporter, potassium channel subfamily U1 (KCNU1, also known as SLO3) has a high level of expression in the testis, and its deletion has been reported to lead to defects in sperm motility and fertility in mouse [3, 4]. In particular, SLO3, first cloned in mouse spermatocytes in 1998, belongs to the high conductance Slo $\mathrm{K}^{+}$channels family and is a sperm-specific potassium channel whose physiological function has been confirmed by whole-cell patch clamping techniques [5]. Similar to SLO1, the calcium and voltage-gated potassium channel, SLO3 consists of a voltage-dependent pore formed by four identical $\alpha$-subunits and a large cytoplasmic C-terminal, which has been proposed to be the ligand-binding sensor [6]. Both the $\mathrm{pH}$ and voltage dependence as well as the properties of the single channel of SLO3 have been well defined [7]. Despite the low sequence conservation of SLO3 among different mammalian species, it displays a high testis-specific expression pattern, located in the main part of the flagellum of sperm in mice $[8,9]$. In addition, SLO3 has been reported to be sensitive to both $\mathrm{pH}$ and the voltage level and to play a role in sperm capacitation, sperm motility, and acrosome reaction. Deletion of the gene coding this ion channel might result in defective sperm activation and fertility in mice $[3,4,10,11]$.

So far, studies on the function of SLO3 have primarily focused on the mouse orthologue, mSLO3. Electrophysiological analysis and genetic deletion studies established that $\mathrm{mSLO} 3$ is a voltage- and $\mathrm{pH}$-gated channel that mediates most $\mathrm{K}^{+}$current in murine sperm. The function of mSLO3 has been shown to be essential for male fertility as Slo3 knockout (KO) mice were found to be infertile, with sperm cells exhibiting a series of functional defects. In humans, it is unknown whether $\mathrm{SLO} 3$ is functionally expressed in the sperm and serves a similar key role for the normal morphology and motility of the sperm and fertilization. Some reports have studied the properties of the hSLO3 channel by using the heterologous expression of hSLO3 channels in Xenopus oocytes [12, 13]. However, the exact functional properties of hSLO3 have not been characterized in human sperm. In this study, we analysed genetic data obtained by whole-exome sequencing (WES) from a large cohort of 105 patients with asthenoteratozoospermia, and identified a single patient carrying a homozygous missense mutation in $S L O 3$. The adult male harbouring the homozygous $\mathrm{SLO} 3$ variant was sterile, and characterized by abnormal sperm morphology and reduced sperm motility. However, intra-cytoplasmic sperm injection (ICSI) treatment using the mutant spermatozoa led to successful fertilization. Therefore, this work provided the first evidence that absence of $\mathrm{SLO} 3$ causes male infertility due to abnormalities in sperm morphology, the mitochondrial sheath, acrosome reaction, and membrane potential of sperm in human.

\section{Materials and methods Subjects}

In this study, we recruited a total of 105 Chinese men with asthenoteratozoospermia from the First Affiliated Hospital of Anhui Medical University. All 105 idiopathic infertile men were diagnosed with primary infertility for $>1$ year. The individuals were recruited according to the guidelines of the World Health Organization (WHO) Laboratory Manual for the Examination and Processing of Human Semen [14]. Individuals with obvious primary ciliary dyskinesia-related symptoms, such as bronchitis, sinusitis, otitis media, or pneumonia, as well as infertility caused by reproductive malformation, drugs, or exposure to gonadotoxic factors, were excluded. Individuals with abnormalities in their karyotype $(46, \mathrm{XY})$ or Y chromosome microdeletions were also excluded. Peripheral whole blood samples were collected from all recruited participants for subsequent genetic analysis. 


\section{Ethical approval}

This study was approved by the ethics committee of the First Affiliated Hospital of Anhui Medical University. Informed consent was obtained from all participants and their family members, as well as from all fertile control male individuals.

\section{Semen parameters and sperm morphological analysis}

Semen samples from men with asthenoteratozoospermia and control subjects were collected via masturbation after 3-7 d of sexual abstinence and measured after liquefaction for $30 \mathrm{~min}$ at $37^{\circ} \mathrm{C}$ in the source laboratories during the routine biological examination of individuals in accordance with the WHO guidelines (5th Edition) $[14,15]$. Analyses of semen volume, sperm concentration, and motility were conducted during routine examination. Sperm morphology was analysed using haematoxylin and eosin (H\&E) staining assay as previously described [16, 17], and 213 spermatozoa to evaluate the percentage of morphologically abnormal spermatozoa, such as small acrosome, swollen midpiece, and coiled flagella. One spermatozoon was classified in only one morphological category according to its major abnormality.

\section{Structural modeling for $\mathrm{SLO} 3$ and its mutants}

The mutant Ile413Phe of SLO3 is located near the Pfam motifs, the effect on protein structure were modelled with homology models. 3D structural model of the SLO3 mutant was carried out using UCSF Chimera software, based on the 4HPF.pdb protein template.

\section{Whole exome sequencing, bioinformatic analysis, and sanger sequencing}

Genomic DNA was extracted from the peripheral blood of participants for WES analysis. Details on the methods used for library construction, WES, and data analysis were previously described [18]. The SLO3 variant identified by WES was further validated by Sanger sequencing using the PCR primers presented in Table S1.

\section{Scanning and transmission electron microscopy}

For the electron microscopy assay, semen samples were prepared in accordance with a protocol previously described [19]. Briefly, for scanning electron microscopy (SEM), samples were sequentially dehydrated using an ascending gradient of ethanol (Shengqiang Medical Technology, Jiangsu, China) and then dried with hexamethyldisilane (HMDS, Sigma-Aldrich, Castle Hill, NSW, Australia). Samples were then air-dried, added dropwise to specimen stubs, sputter coated, and examined using field emission SEM (Nova Nano 450, Thermo Fisher Scientific Inc., USA). For transmission electron microscopy (TEM), samples were fixed with 2.5\% osmium tetroxide (Sigma-Aldrich, Castle Hill, NSW, Australia) and sequentially dehydrated using graded ethanol (50, 70, 90, and 100\%) and 100\% acetone (Sigma-Aldrich, Castle Hill, NSW, Australia). Samples were then infiltrated with acetone and SPI-Chem resin and embedded with Epon 812 (SPI\#02659-AB, Structure Probe, USA). Subsequently, samples were sliced using an ultra-microtome (UC7, LEICA EM, Germany) and stained with uranyl acetate (\#19481, Ted Pella, Inc., Redding, CA) and lead citrate (\#19312, Ted Pella, Inc., Redding, CA). Cryoelectron microscopy (TecnaiG2 Spirit $120 \mathrm{kV}$, FEI, Netherlands) was used for image capturing.

\section{Quantitative real-time PCR and western blotting}

Total RNA from human spermatozoa was isolated using the TRIzol Reagent (Invitrogen, Carlsbad, CA 92008 USA) and transcribed into cDNA using the PrimeScript RT Reagent Kit (Takara, Shiga, Japan) according to the manufacturer's protocol. The obtained cDNAs were used as templates for subsequent quantitative real-time PCR conducted using the Light Cycler 480 SYBR Green I Master (Roche, Switzerland, Germany). $\beta$-actin was used as an internal control. The assays were repeated thrice. Primers used were listed in Table S2.

Proteins from human sperm samples for immunoblotting were extracted using the Mem-PER ${ }^{\mathrm{TM}}$ Plus Membrane Protein Extraction Kit $(89,842$, Thermo Fisher Scientific Inc., USA) according to the manufacturer's instructions. Briefly, washed sperm samples were suspended in $0.5 \mathrm{~mL}$ permeabilization buffer, vortexed, and incubated for $10 \mathrm{~min}$ at $4{ }^{\circ} \mathrm{C}$ with constant mixing. Permeabilized sperm samples were then centrifuged for $15 \mathrm{~min}$ at $16000 \mathrm{~g}$ and the supernatant containing cytosolic proteins was transferred to a new tube. The pellet was resuspended in $0.5 \mathrm{~mL}$ solubilization buffer and incubated for $30 \mathrm{~min}$ at $4{ }^{\circ} \mathrm{C}$ with mixing. Finally, resuspended samples were centrifuged at $16000 \mathrm{~g}$ for $15 \mathrm{~min}$ at $4^{\circ} \mathrm{C}$, and the supernatant containing solubilized membranes was collected and heated at $100^{\circ} \mathrm{C}$ for $15 \mathrm{~min}$. Lysates were separated on 10\% SDS-PAGE gels and transferred onto polyvinylidene fluoride (Pall Corporation, New York, NY, USA) membranes. Membranes were blocked in 5\% non-fat milk diluted with TBST (TBS-0.1\% Tween-20) for $1 \mathrm{~h}$ at $25^{\circ} \mathrm{C}$. Membranes were then immunoblotted using the following primary antibodies: rabbit polyclonal anti-LRRC52 (1:1000; PA5-107159, Invitrogen, Carlsbad, CA 92008 USA), rabbit polyclonal anti-CatSper1 (1:1000; DF9349, Affinity Biosciences, Beijing, China), rabbit polyclonal 
anti- $\mathrm{Na}^{+} / \mathrm{K}^{+}$-ATPase $\alpha 1$ (1:2000; ABP51894, Abbkine, China), anti-HSP60 antibody (1:1000; ab13532, Abcam, Cambridge, UK), and HRP-conjugated $\beta$-actin (1:2000; HRP-60008, Proteintech, Rosemont, IL, USA), at $4{ }^{\circ} \mathrm{C}$ overnight. Signals were detected using the ECL Prime Western Blotting Detection Reagent (GE Healthcare, Beijing, China). Images were acquired using a CS analyser system (5200, Tanon, Shanghai, China). The $\mathrm{Na}^{+}$/ $\mathrm{K}^{+}$-ATPase $\alpha 1$ or $\beta$-actin reference proteins were used as loading control.

\section{Generation of polyclonal anti-SLO3 antibody}

SLO3 polyclonal antibodies were generated by ABclonal Biotechnology in New Zealand rabbits using the 11201134 and 1156-1170 polypeptides of the human SLO3 protein (ENSP00000382770) as antigens. Briefly, the cDNA encoding these epitopes was cloned into a pET28a expression vector, and the His-tagged fusion protein was expressed in Escherichia coli. The purified recombinant protein was used to generate polyclonal antisera in female New Zealand rabbits. Sequences of the peptides used were as follows: 1. SYQPRTNSLSFPKQ 2. KENERKTSDEVYDED.

\section{Immunofluorescence assays}

Immunofluorescence staining of sperm samples was performed as previously described [20]. Briefly, sperm samples were washed twice with phosphate buffer saline (PBS), fixed in $4 \%$ paraformaldehyde (Sigma-Aldrich, Castle Hill, NSW, Australia) at $4{ }^{\circ} \mathrm{C}$ overnight, and mounted on slides pre-treated with poly-L-lysine (Sigma-Aldrich, Castle Hill, NSW, Australia). Slides were incubated with primary antibodies (SLO3 (1:200, ABclonal Biotechnology, China), PLC$\zeta 1$ (1:100, pab0367-P, covalab, USA), LRRC52 (1:500, PA5-107159, Invitrogen, Carlsbad, USA), CatSper1 (1:200; DF9349, Affinity Biosciences, Beijing, China), HSP60 (1:500; ab13532, Abcam, Cambridge, UK), acetylated alpha-tubulin (1:1000, mAb\#5335, Cell Signaling Technology, Massachusetts, USA)) and PNA (1:500, RL-1072, VectorLabs, California, USA) overnight at $4{ }^{\circ} \mathrm{C}$. After washing with PBS, slides were incubated with highly cross-adsorbed secondary Alexa Fluor 488 anti-mouse IgG (1:500, 34106ES60, Yeasen Biotechnology, USA) and Alexa Fluor 594 anti-rabbit IgG antibodies (1:500, 111-585-003, Jackson ImmunoResearch Inc., USA) for $1 \mathrm{~h}$ at $37^{\circ} \mathrm{C}$ and subjected to Hoechst (1:1000, 62,249, Thermo Fisher Scientific Inc., USA) nuclear labelling for $2 \mathrm{~h}$ at $37^{\circ} \mathrm{C}$. Images were captured using an LSM 800 confocal microscope (CarlZeiss AG, Germany).

\section{Detection of mitochondrial membrane potential by flow cytometry acquisition}

Measurements of mitochondrial membrane potential (MMP) were performed using the lipophilic cationic dye $5,5^{\prime}, 6,6^{\prime}$-tetrachloro-1, 1', 3, 3'-tetraethylbenzimidazolylcarbocyanineiodide (JC-1) according to the manufacturer's instructions (Invitrogen). Briefly, approximately 1 million sperm cells were washed with PBS, followed by incubation with $5 \mu \mathrm{M} \mathrm{JC}-1$ working solution for $15 \mathrm{~min}$ at $37^{\circ} \mathrm{C}$. Samples were then washed and analysed by flow cytometry. For further analysis of all cytometric experiments, the debris was gated out based on light scattering measurements. For each analysis, at least 50,000 sperm cells were re-examined. All experiments were performed on a BD FACSVerse ${ }^{\mathrm{TM}}$ Flow Cytometer (BD Biosciences, USA). Flow cytometry acquisition for JC-1-stained sperm cells was performed through FL1 for green and FL2 for red fluorescence. At high MMP, JC-1 forms J-aggregates inside the mitochondria emitting red fluorescence, whereas in a low MMP state, it remains in the monomer form emitting green fluorescence.

\section{Measurement of sperm membrane potential by flow cytometry}

Sperm samples were washed twice with PBS. Pellets were then resuspended in Whitten's HEPES-buffered media [(in mM): $135 \mathrm{NaCl}, 5 \mathrm{KCl}, 2 \mathrm{CaCl}_{2}, 1 \mathrm{MgSO}_{4}, 20$ HEPES, 5 glucose, 10 lactic acid, 1 Na-pyruvate] with or without $25 \mathrm{mM} \mathrm{NaHCO}$ and $5 \mathrm{~g} / \mathrm{L} \mathrm{BSA}$ and incubated with $1 \mu \mathrm{M}$ potential-sensitive dye $3,3^{\prime}$-dipropylthiocabocyanine iodine (DiSC3, Sigma-Aldrich, Castle Hill, NSW, Australia) for $8 \mathrm{~min}$ at $37^{\circ} \mathrm{C}$. After incubation, PI (Sigma-Aldrich, Castle Hill, NSW, Australia) was added and incubated for further $3 \mathrm{~min}$ at $37^{\circ} \mathrm{C}$. Before assaying the sperm using flow cytometry, $500 \mathrm{nM}$ carbonyl cyanide $\mathrm{m}$-chlorophenylhydrazone (CCCP, Sigma-Aldrich, Castle Hill, NSW, Australia) was added in sperm suspensions and incubated at $37^{\circ} \mathrm{C}$ for $2 \mathrm{~min}$ to dissipate the mitochondrial membrane potential. Analyses were conducted using a FACSVerse ${ }^{\mathrm{TM}}$ Flow Cytometer. Orange fluorescence from DiSC3-positive cells was detected at 600-700 nm and PI was detected at 500-560. Data were analyzed using FACS Diva and FlowJo software (Tree Star 9.3.3) as previously described [13]. Cell debris, doublets and aggregates were excluded from analysis based on a dual parameter dot plot, in which pulse signal (signal high; FSC-H; $y$-axis) versus signal area (FSC-A; $\mathrm{x}$-axis) was displayed.

\section{ICSI procedures}

The female partner of individual with SLO3 mutation had undergone a long protocol pituitary downregulation 
using GnRH agonist (Triptorelin, Diphereline $3.75 \mathrm{mg}$, Ipsen Pharma Biotech) and control ovarian hyperstimulation by recombinant FSH (Gonal-F; Serono) Oestradiol plasma levels and follicle growth were monitored every 2 days and human chorionic gonadotrophin (HCG, Livzon Pharmaceutical) was administered when three or more than follicles reached $18 \mathrm{~mm}$ in diameter. $36 \mathrm{~h}$ after HCG injection, 15 mature oocytes (MII) were retrieved for ICSI. Spermatozoa were prepared by discontinuous density gradient centrifugation and the resulting suspension was diluted in $10 \mu \mathrm{l}$ drops of polyvinyl pyrolidine (PVP) covered with oil. Subsequently, metaphase II stage oocytes and motile sperm were selected for ICSI using a micromanipulator system (Olympus, Japan). Eighteen to $19 \mathrm{~h}$ later, the fertilized oocytes were assessed and cultured in cleavage medium (Cook, USA) in an incubator with an environment of $37^{\circ} \mathrm{C}, 5 \% \mathrm{O}_{2}, 6 \% \mathrm{CO}_{2}$, and $89 \%$ $\mathrm{N}_{2}$ until day 3 after fertilization. Then, the evaluated embryos were transferred to blastocyst medium (Cook, USA) and incubated to day 5 or day 6 . According to the scoring system of Gardner and Schoolcraft [21], we obtained three day- 5 blastocysts (4AB, $4 \mathrm{BB}$ and $3 \mathrm{BB}$ ) and nine day- 6 blastocysts $(4 \mathrm{BA}, 4 \mathrm{BB}, 4 \mathrm{BC}, 3 \mathrm{BB} * 2$ and $3 C C^{*} 3$ ), and nine blastocysts (3CC*3 poor blastocysts were discarded) were cryopreserved to prevent ovarian hyperstimulation syndrome (OHSS). After 6 months, two day- 5 blastocysts were thawed and transferred in successive artificial cycles and a single foetal heart beat was detected via ultrasound after 28 days.

\section{Statistical analyses}

All data are representative of at least three independent experiments, and GraphPad Prism (GraphPad Software, San Diego, CA, USA) was used to perform the statistical analysis. Differences were analyzed by Student's t-tests when comparing experimental groups, and $P$-values $<0.05$ were considered significant.

\section{Results \\ SLO3 is involved in human male infertility with asthenoteratozoospermia}

We performed WES on a cohort of 105 individuals with severe asthenoteratozoospermia. After applying stringent filters criteria according to our previously described protocol, we identified a single individual (A-132) carrying a homozygous mutation in SLO3 (GenBank: NM_001031836.3, c.1237A > T: p.Ile413Phe). The SLO3 mutation was confirmed by Sanger sequencing (Fig. 1A). The subject A-132 from a consanguineous family, and presented with severely reduced sperm motility and a comparatively low sperm count $\left(12.7 \times 10^{6} / \mathrm{mL}\right)$ (Table 2, Fig. 1A). We noticed that the allele frequency of the $S L O 3$ variant was $<1 \%$ in any database, including
1000 Genomes Project and gnomAD (v2.1.1 with 141,456 samples). Moreover, the PolyPhen-2, SIFT, and MutationTaster tools predicted it to be deleterious (Table 1).

The $\mathrm{SLO} 3$ (also known as KCNU1) gene is located on human chromosome 8 and contains 27 exons encoding a predicted 1149 -amino acid potassium channel protein, specifically expressed in human spermatozoa. The SLO family channels are composed of 4 transmembrane voltage sensor domains (VSDs) surrounding a central pore gate domain (PGD), and a large cytosolic domain known as the gating ring. The gating ring is assembled from the regulators of $\mathrm{K}^{+}$conductance (RCK1 and RCK2) domains of each of the 4 subunits, forming a ring with a central opening. The RCK domain determines the closed vs open conformational state of the gating ring and transduces the effects of any factor interacting with the gating ring to the PGD. Interestingly, the residue in the RCK domain of the SLO3 protein affected by the $\mathrm{SLO} 3$ missense variant p. Ile413Phe is highly conserved in SLO3 orthologues (Fig. 1B). Further analysis of protein structural modelling using online bioinformatic tools revealed the severe effects of this amino acid-substituting mutation on the structure of the SLO3 protein, indicating that this mutation introduces an amino acid with different properties, which may disturb this domain and abolish its function (Fig. 1C).

\section{Morphological abnormalities in sperm heads and mid-piece flagella of the man harbouring the homozygous SLO3 variant}

We examined sperm morphological and ultrastructural changes in sperm of SLO3 mutant subject. Compared with the sperm from a fertile control individual, the spermatozoa from the individual harbouring the $\mathrm{SLO} 3$ variant displayed a high rate of head malformations, in particular, an abnormal acrosomal region, indicating a defect in acrosome formation. In addition, we observed that sperm tails displayed swollen midpieces or shorter principal pieces of highly irregular width, indicating poorly assembled mitochondrial sheaths (Fig. 2A and Table 2). When observed by SEM, we found that spermatozoa of unaffected controls exhibited a smooth, regularly contoured, oval-shaped head and a long flagellum with a clearly defined midpiece, whereas most spermatozoa from the individual with the $\mathrm{SLO} 3$ variant exhibited severe morphological defects (Fig. 2B), such as swollen midpieces (II and V), coil-shaped flagella (III), small acrosome (IV), absence of acrosome in combination with defective midpiece (VI and VII), and insufficient chromatin condensation (VIII).

To better understand the nature of these head and flagella defects observed in the spermatozoa with the SLO3 mutation, we analysed their ultrastructure. We 


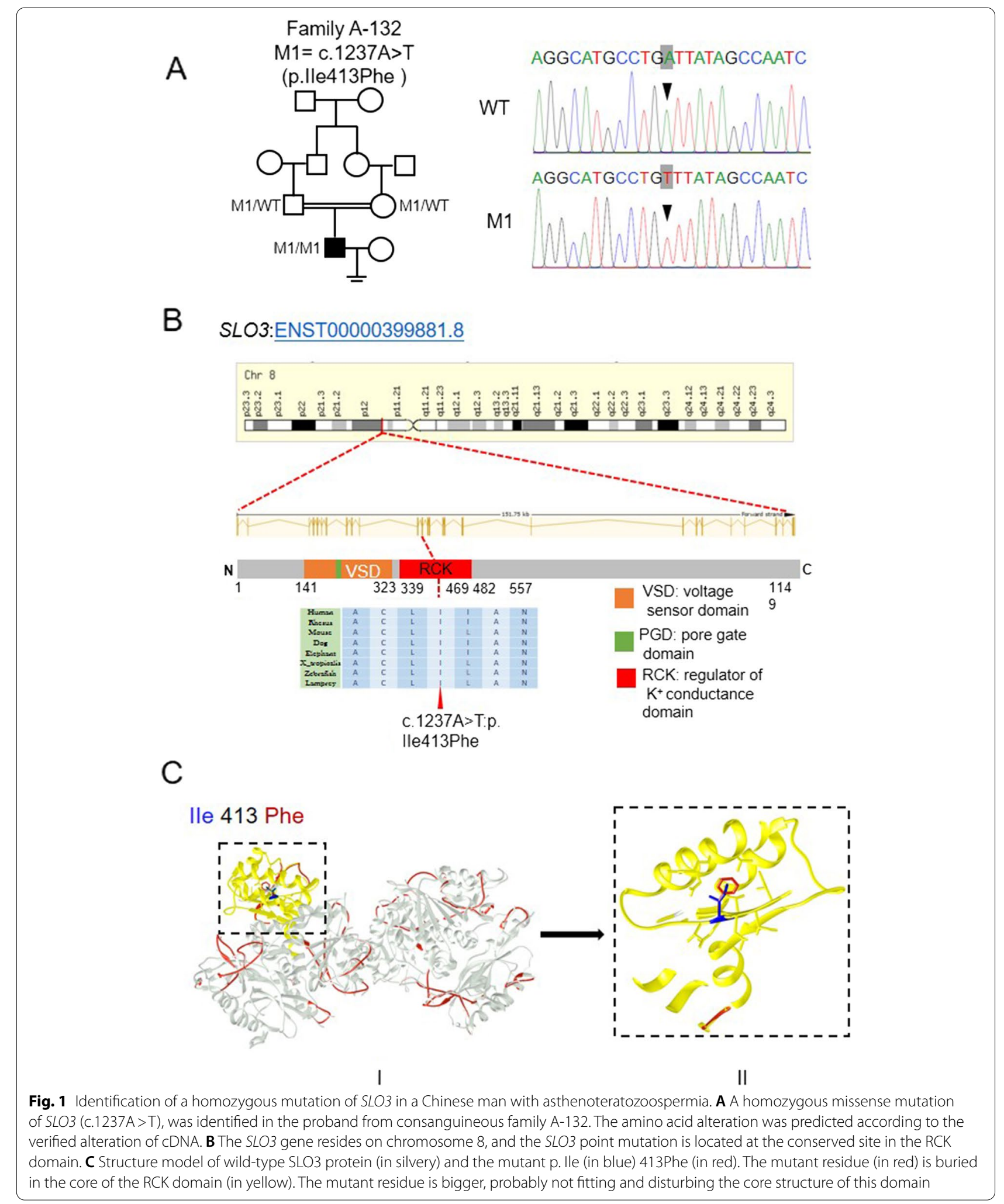


Table 1 Identification of Homozygous SLO3 Variant in the Subject with Severe Asthenoteratozoospermia

\begin{tabular}{ll}
\hline SLO3 Variant Information & Subject A-132 \\
\hline CDNA alteration & C.1237A>T \\
Variant allele & Homozygous \\
Protein alteration & p. lle413Phe \\
Variant type & Missense variant \\
Allele Frequency in Human Populations & \\
$\quad$ IKGP & 0.0000122861 \\
East Asians in gnomAD & 0.0048076 \\
All individuals in gnomAD & 0.00004215 \\
Function Prediction & \\
$\quad$ SIFT & damaging \\
PolyPhen-2 & damaging \\
MutationTaster & disease causing
\end{tabular}

NCBI reference sequence accession number of SLO3 is NM_001008723.2 Abbreviations: 1KGP 1000 Genomes Project, gnomAD Genome Aggregation Database

accordingly found that their acrosomes were either absent or morphologically defective (Fig. 2C). We also noticed that most acrosomes were quite thin, showing highly diminished contents with barely recognizable inner and outer acrosomal membranes. Furthermore, a higher incidence of insufficient chromatin condensation was also observed in the spermatozoa of the affected individual compared with those of controls. The longitudinal sections of the flagellar mid-piece of spermatozoa in the individual harbouring the homozygous SLO3 variant showed a seriously disorganized or short mitochondrial sheath compared with those of control (Fig. 2C). However, we noticed that in cross-sections, the arrangements of ODF, DMT, and CP were not significantly different (Fig. 2D). Indeed, many mutant spermatozoa had a coil-shaped flagella that might have been associated with deficient osmoregulation and volume control, a condition expected to result from the mutation-induced ablation of the ion channel.

To further characterize the molecular defects induced by the $S L O 3$ mutation in human sperm, we examined the expression of SLO3 mRNA and protein in sperm samples from control and individuals harbouring the $S L O 3$ variant. As shown in Fig. 3A, the SLO3 mRNA was significantly $(P<0.001)$ reduced in spermatozoa from the man harbouring the SLO3 variant, as well as the protein expression level (Fig. $3 \mathrm{~B}$ and $\mathrm{C}$ ), indicating that the observed sperm defects were probably induced by the deficiency in SLO3.

\section{SLO3 deficiency affected the functions of acrosome and mitochondria}

The ultrastructure analysis of spermatozoa from the individual harbouring the SLO3 variant indicated that SLO3 might play an essential role in acrosome formation and mitochondrial sheath assembly. To investigate the effect of the SLO3 variant on the spermatozoa acrosome state, we evaluated the percentage of spontaneous acrosomereacted cells using PSA-FITC binding. We found a significant increase (Student's $t$-test; $P<0.001$ ) in the rate of acrosome reaction in spermatozoa of the man harbouring the SLO3 variant compared with that of controls (Fig. 4A and $B$ ). In addition, we also observed that most spermatozoa from controls presented a highly stained PNA in acrosomal region, whereas the signal was absent in most spermatozoa from the SLO3 mutant individual. Moreover, the localization patterns of PLC $\zeta 1$ were located in the acrosomal region in most spermatozoa from control individuals (Fig. 4C). However, spermatozoa from the subject harbouring the $S L O 3$ variant showed a predominant base of the neck and middle-piece of the tail localization pattern, indicating that both the formation of the acrosome and the localization pattern of PLC $\zeta 1$ were influenced by the SLO3 deficiency.

\footnotetext{
(See figure on next page.)

Fig. 2 Sperm morphology and ultrastructure analyses in the spermatozoa from SLO3-mutated subject A-132. A Light microscopy analysis of spermatozoa from control (i) and the individual harbouring the SLO3 variant. Most spermatozoa from the individual harbouring the SLO3 variant have small acrosomal heads, swollen midpieces, and coil-shaped flagella. B SEM analyses of sperm cells from a fertile control and the SLO3-mutated individual (II-VIII). Spermatozoa of normal controls exhibit a smooth, regularly contoured, oval-shaped head and a flagellum with a clearly defined midpiece, whereas most sperm from the SLO3 mutant individual exhibit severe morphological defects (IIIVIII), such as swollen midpieces (II), coil-shaped flagella (III), small acrosome (IV), and absence of acrosome in combination with a defective midpiece (V-VIII). CTEM analyses of sperm cells from a fertile control and the SLO3-mutated individual. The longitudinal sections of spermatozoa from control and A-132. Sperm tails with poorly assembled mitochondria (II-red arrow) or a cytoplasmic mass containing different components of the flagellum (III-red arrow) are observed. The acrosome is thin or broken with unidentifiable acrosomal membranes (red asterisk) along with misshapen heads; chromatin condensation appears abnormal (number sign). D Cross-sections of the mid-piece, principal piece, and end piece of the flagella in a control individual show the typical " $9+2$ " microtubule structure, including 9 peripheral microtubule doublets paired with 9 outer dense fibres and the central pair of microtubules, surrounded by the organized mitochondrial sheath or fibrous sheath. Ultrastructures of sperm from the SLO3-mutated individual are comparable to those from control. Scale bars: $10 \mu \mathrm{m}$ (A), $1 \mu \mathrm{m}$ (B), $2 \mu \mathrm{m}$ (C), and $200 \mu \mathrm{m}$ (D)
} 


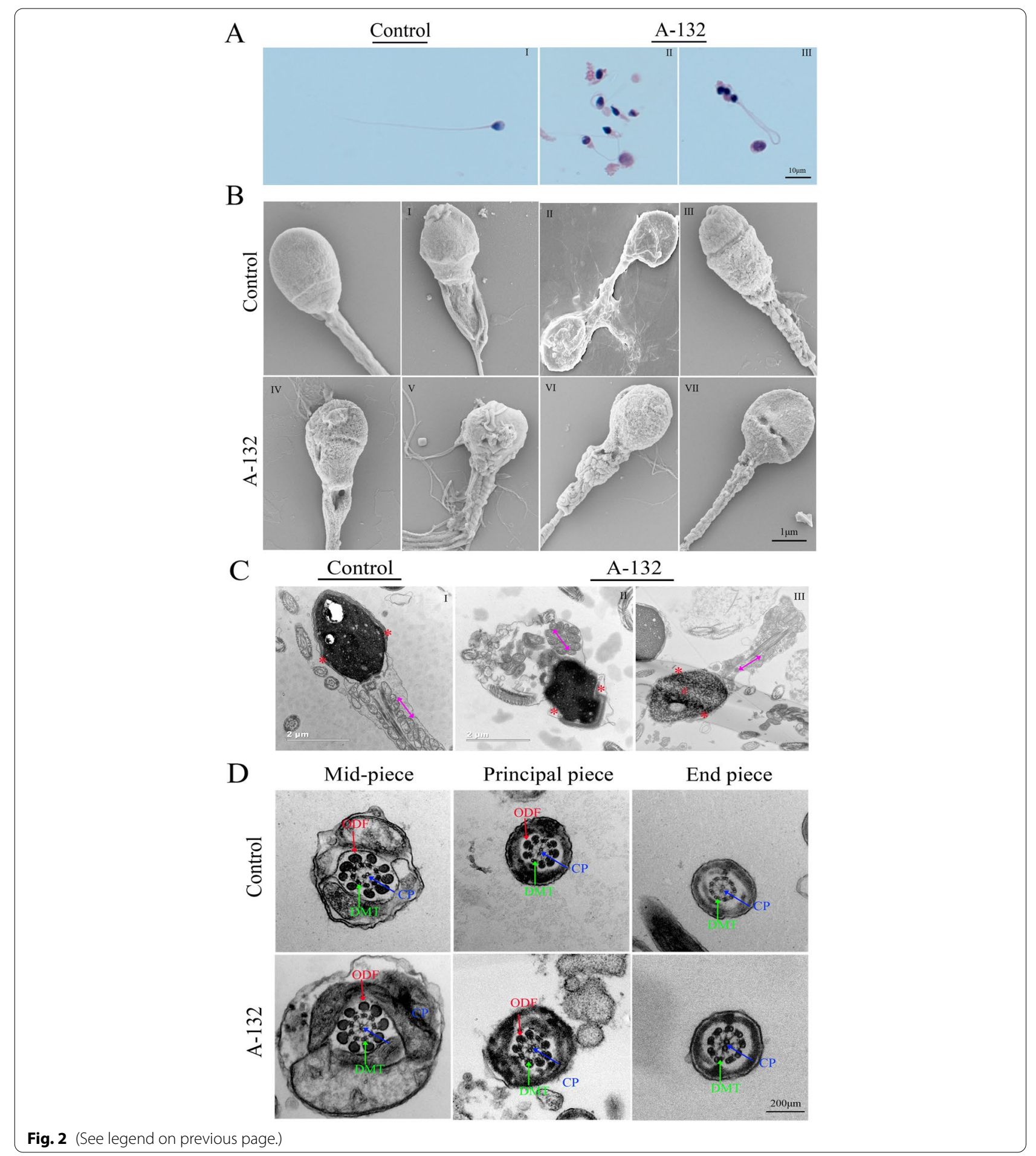

Furthermore, we investigated the effect of $\mathrm{SLO} 3$ deficiency on mitochondrial function. As shown in Fig. 5A, compared with control, the mitochondrial membrane potential was drastically decreased in spermatozoa from the man harbouring the $\mathrm{SLO} 3$ variant. Subsequently, we examined the expression of HSP60 in the spermatozoa of the affected individual with that of healthy controls. We found that the expression of HSP60 was significantly reduced in the mitochondrial sheath components (Fig. 5B and C), in consistency with the sperm motility 
Table 2 Semen Parameters and Sperm Morphology of the SLO3mutated Man

\begin{tabular}{lll}
\hline Subject & A-132 & Reference Values \\
\hline Semen Parameters & & \\
$\quad$ Semen Volume $(\mathrm{mL})$ & 4.3 & $>1.5^{\mathrm{a}}$ \\
Concentration $\left(10^{6} / \mathrm{mL}\right)$ & $\mathbf{1 2 . 7}$ & $>15.0^{\mathrm{a}}$ \\
Motility (\%) & $\mathbf{3 1 . 9}$ & $>40.0^{\mathrm{a}}$ \\
Progressive motility (\%) & $\mathbf{4 . 8}$ & $>32.0^{\mathrm{a}}$ \\
Sperm Morphology & & \\
Normal sperm (\%) & $\mathbf{2 . 3}(5 / 215)$ & $>4.0^{\mathrm{a}}$ \\
Small acrosome (\%) & $\mathbf{6 1 . 4}(132 / 215)$ & $<5.0^{\mathrm{b}}$ \\
Abnormal midpieces (\%) & $\mathbf{3 5 . 3}(76 / 215)$ & $<11.4^{\mathrm{c}}$ \\
Coiled flagella (\%) & $\mathbf{5 0 . 2}(108 / 215)$ & $<17.0^{\mathrm{b}}$ \\
Abnormal sperm (\%) & $\mathbf{9 7 . 7}$ & $<96^{\mathrm{a}}$ \\
\hline
\end{tabular}

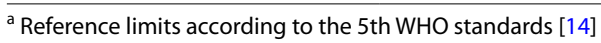

${ }^{b}$ Reference limits according to the distribution range of morphologically normal spermatozoa observed in 926 fertile subjects [17]

' Reference limits according to the distribution range of morphologically normal spermatozoa observed in 10 fertile subjects

At least 200 spermatozoa were observed for morphology analysis

and the TEM results for spermatozoa of the affected individual. These results demonstrated that $\mathrm{SLO} 3$ plays a key role in spermiogenesis by upholding the integrity of acrosomal and mitochondrial sheath components.

The hyperpolarization of sperm from individual harbouring SLO3 variant was decreased during capacitation

In mouse sperm, deletion of the Slo3 gene abolished alkalization-activated $\mathrm{K}^{+}$current [4]. Thus, we wondered whether the SLO3 homozygous mutant would alter the membrane potential of sperm during capacitation. Consistently, we found that the hyperpolarization of sperm from individual harbouring $\mathrm{SLO} 3$ variant was decreased significantly during capacitation (Fig. 6A). To understand the molecular mechanism of the observed lack of membrane potential after capacitation in the $\mathrm{SLO} 3$ mutant sperm, we examined the expression of the SLO3 auxiliary subunit LRRC52 in sperm cells from control and the SLO3-mutant individual Compared with normal controls (Fig. 6B and C), we noticed that the expression of LRRC52 was significantly decreased in sperm cells from the SLO3-mutant individual. Furthermore, we also investigated the sperm-specific change in CatSper expression in the spermatozoa of the SLO3 mutated individual. We specifically observed that immunostaining of CatSper1 in the spermatozoa from the individual harbouring the SLO3 variant was comparable to that observed in control sperm (Fig. 6B and D), suggesting that the $\mathrm{Ca}^{2+}$-activated $\mathrm{K}^{+}$currents were controlled by LRRC52 and not CatSper1.
SLO3 deficiency-related male infertility could be rescued by intracytoplasmic sperm injection treatment

It has been previously reported that $S l o 3^{-/}$male mice are infertile, but $\mathrm{Slo3}^{-/-}$sperm exhibits some fertility when utilized in vitro fertilization assays. In addition, ICSI has been suggested as an effective way to circumvent the physical limitations experienced by asthenoteratozoospermia sperm in our hospital. The individual with the $\mathrm{SLO} 3$ variant was 29 years old at treatment, while his partner was 34 years old. As a couple, they underwent 1 ovarian stimulation cycle, with the number of metaphase II oocytes retrieved and injected being 15 . We observed that the rates of the fertilization, cleavage, 8-cell and blastocyst formation ware comparable to those in OAT controls (Table 3). Consecutively, 9 blastocysts were frozen. Six months later, 2 blastocysts were transferred and a single foetal heart beat was detected via ultrasound, with the pregnancy being currently ongoing. Therefore, our study indicated that male infertility caused by a homozygous SLO3 variant could be rescued by ICSI treatment.

\section{Discussion}

To the best of our knowledge, we demonstrated for the first time that the presence of a homozygous mutation of SLO3 could induce severe morphological defects in the head and midpiece of human spermatozoa, together with a deficiency in the acrosome reaction and impaired progressive motility in sperm. The SLO3 protein localizes in the flagellum and is especially concentrated at the flagellar midpiece of spermatozoa, possibly playing an important role in mitochondrial sheath assembly. In addition, when observed by light microscopy and SEM, most spermatozoa from the individual harbouring the $\mathrm{SLO} 3$ variant exhibited angular/bent tails compared with those of healthy controls, consistent with the observations in $\mathrm{Slo}^{-1-}$ mouse sperm [4]. However, in cross-sections, the arrangements of ODF, DMT, and CP were not significantly different, which might have been associated with a deficient osmoregulation and volume control in sperm, a condition probably resulting from the mutation-induced ablation of a major ion channel [4].

The eukaryotic SLO1 and SLO3 proteins exhibit a high amino acid sequence identity ( $42 \%$ identity), and, in fact, sequence analysis has suggested that $\mathrm{SLO} 3$ evolved from a duplication of the $S L O 1$ gene $[6,22]$. However, SLO1 and SLO3 channels share a unique property among the extended family of voltage-gated $\mathrm{K}^{+}$channels in that their opening requires the synergistic action of membrane depolarization and specific intracellular cues [6]. The $S L O 1$ gene is expressed in excitable cells, such as neurons or muscles [5]. Whereas $S L O 3$, which is exclusively expressed in mammalian 


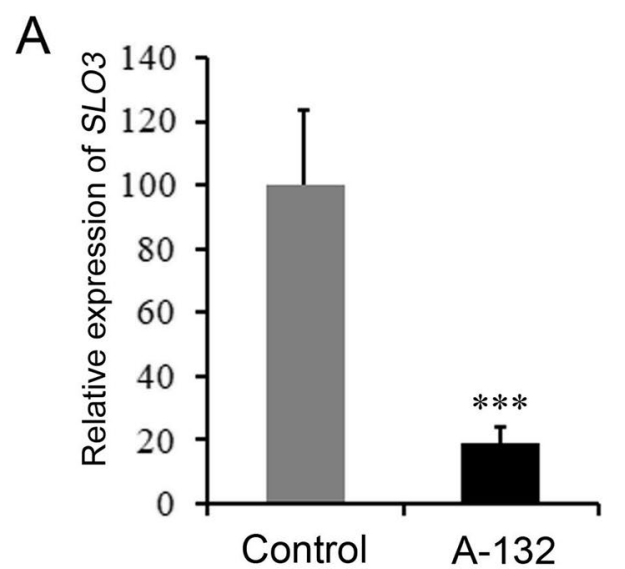

B
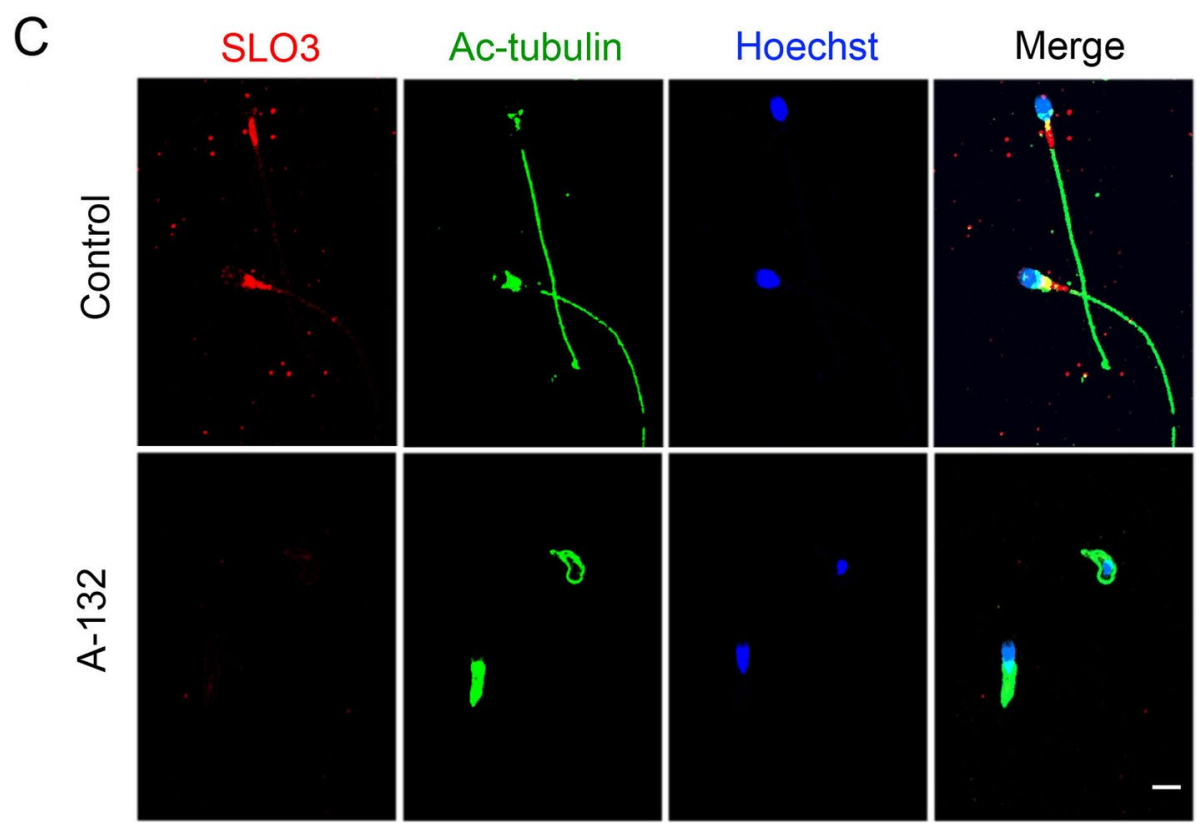

Fig. 3 Expression analysis of SLO3 mRNA and protein and localization of the SLO3 protein in sperm flagella. A Quantitative real-time PCR analysis of the expression of SLO3 mRNA in the spermatozoa from normal control and the SLO3-mutated individual. Compared with control, the expression of SLO3 mRNA is significantly reduced in the sperm from the SLO3-mutated individual. $\beta$-actin was used as an internal control. Data are presented as the mean \pm SEM. ${ }^{* * *} P<0.001$; Student's t-test. B Immunoblotting of sperm lysates from a normal control and the SLO3-mutated individual using an anti-SLO3 antibody. $\mathrm{Na}^{+} / \mathrm{K}^{+}$-ATPase 11 was used as loading control. C Representative images of spermatozoa from controls and from the SLO3-mutated individual stained with anti-SLO3 antibody, anti-Ac-tubulin antibody, and Hoechst. SLO3 staining is concentrated at the mid-piece of sperm flagella and faintly along the flagella in the fertile control but is significantly reduced in the sperm flagella of the individual harbouring the SLO3 mutation. Scale bar, $20 \mu \mathrm{m}$

\section{(See figure on next page.)}

Fig. 4 The acrosome reaction and expression patterns of acrosome are changed in the spermatozoa from SLO3-mutant man. A Influence of the SLO3 mutation on spontaneous acrosome reaction. Staining with PSA-FITC: acrosome reacted spermatozoa (red asterisk); non-acrosome reacted spermatozoa (red arrowhead). B Percentage of spontaneous acrosome reaction. Data are presented as the mean \pm SEM. Bars represent standard error. Significant differences, ${ }^{* *} P<0.001$; Student's $t$-test. C Peanut agglutinin (PNA) staining conjugated to rhodamine for the localization patterns of spermatozoa membrane galactose. $\mathbf{D}$ Localization patterns of PLC 11 in normal and mutant sperm. Representative images of spermatozoa from controls and the SLO3-mutated individual stained with anti-PLC $\zeta 1$ antibody, anti-Ac-tubulin antibody, and Hoechst. PLC 1 staining is concentrated at the acrosomal region in control sperm, but absent in most mutant sperm, Scale bars: $20 \mu \mathrm{m}$ 


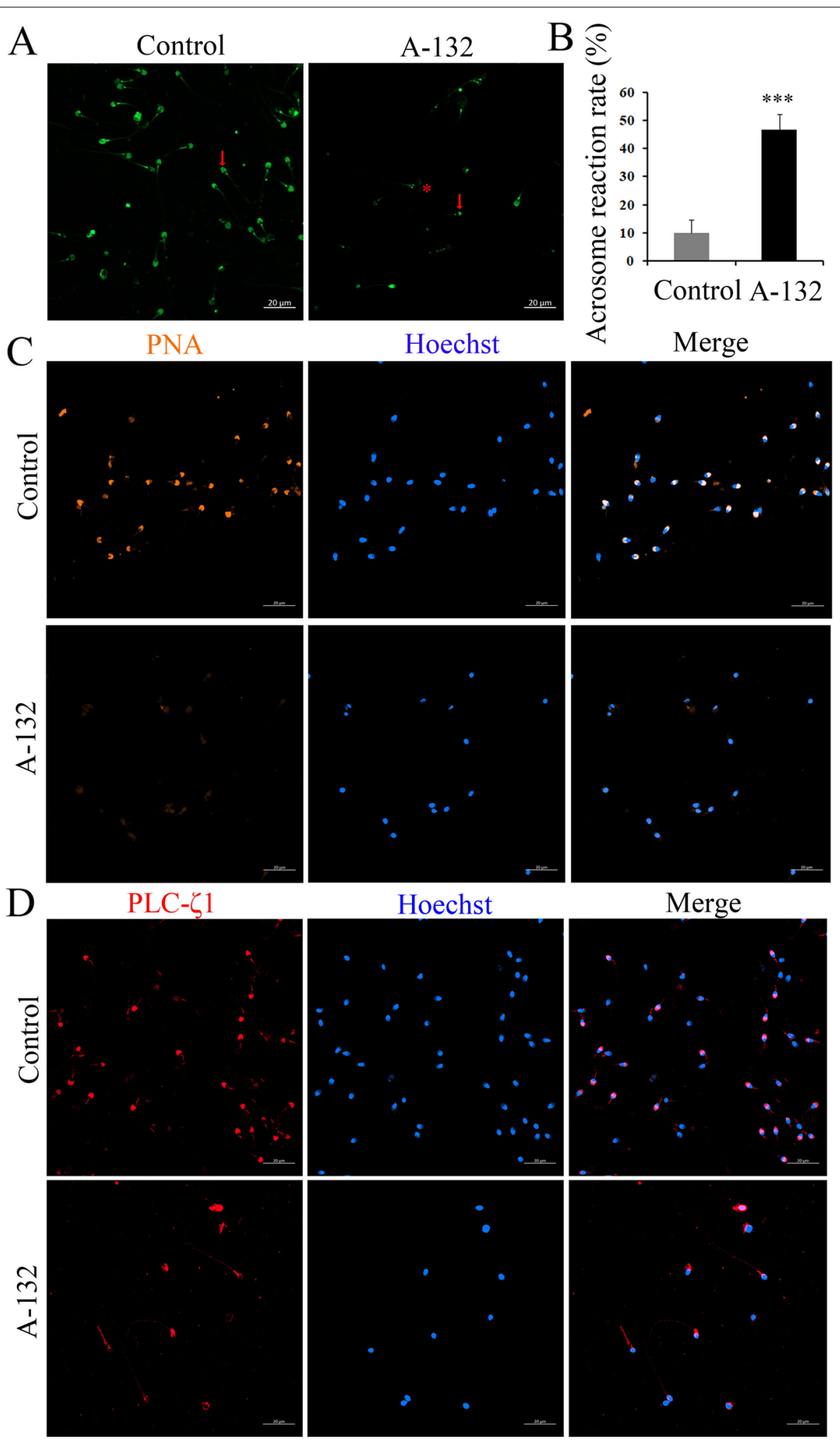

Fig. 4 (See legend on previous page.) 

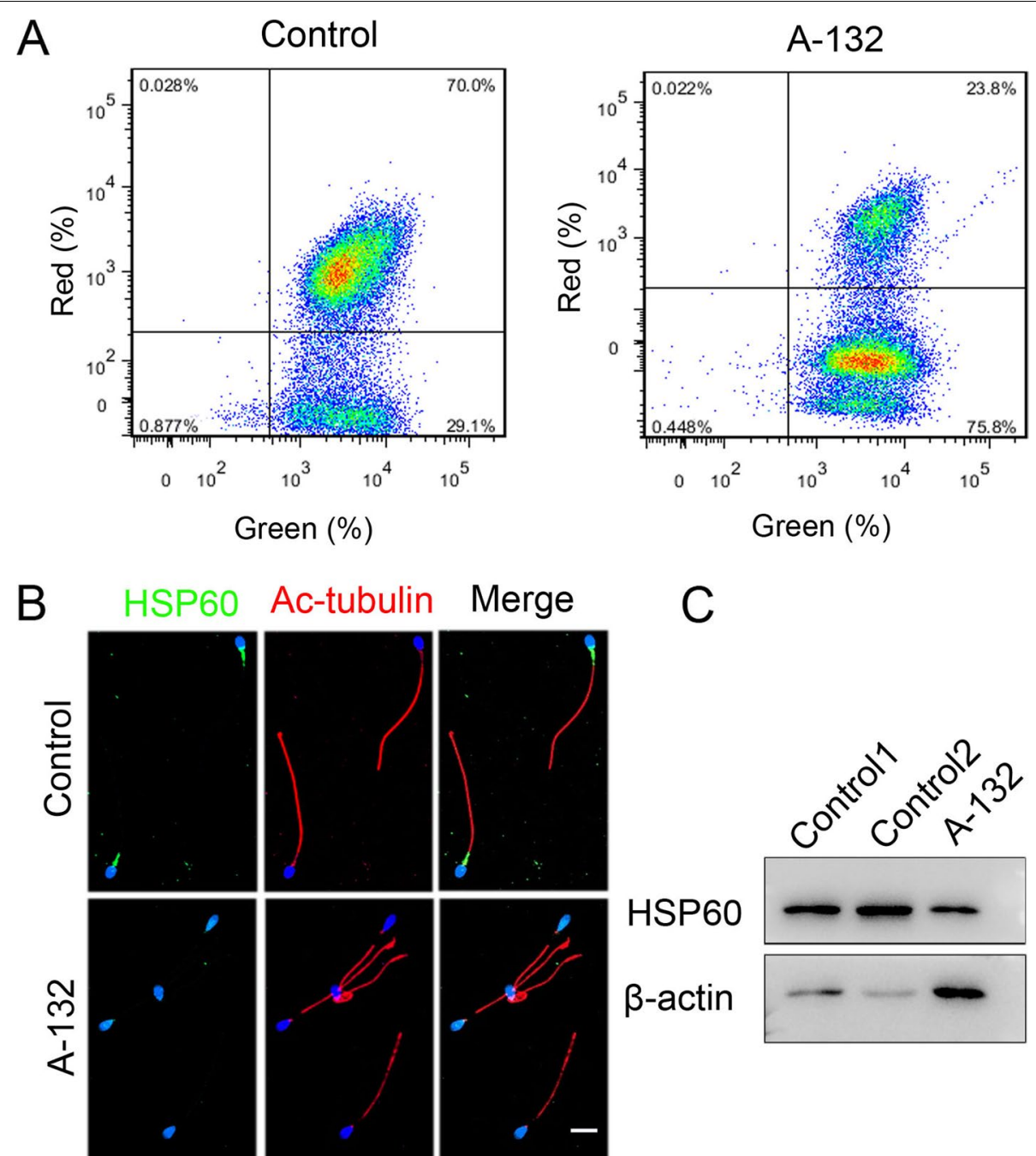

Fig. 5 Effects of SLO3 deficiency on mitochondrial function. A Influence of the SLO3 mutation on mitochondrial membrane potential (MMP). Assessment of mitochondrial membrane potential by flow cytometry acquisition for JC-1-stained (a marker of MMP) cells was performed through FL1 for green and FL2 for red fluorescence. MMP is significantly reduced in mutant sperm compared with control. B Immunoblotting of sperm lysates from controls and the SLO3-mutated individual using the anti-HSP60 antibody. $\beta$-actin was used as loading control. C The expression of HSP60 is almost diminished in SLO3 mutant spermatozoa. HSP60 staining is present in the flagellar midpiece of spermatozoa from normal controls, but significantly reduced in spermatozoa from A-132. Scale bars, $20 \mu \mathrm{m}$

sperm, is a voltage- and $\mathrm{pH}$-gated channel that mediates most $\mathrm{K}^{+}$current in murine sperm essential to male fertility and is activated by an increase in intracellular $\mathrm{pH}[3,23]$. In humans, the exact functional properties of hSLO3 have not been characterized. By heterologously expressing human $\mathrm{SLO} 3$ in Xenopus oocytes, MacKinnon et al. found that the $\mathrm{pH}$ sensitivities of hSLO3 and mSLO3 were comparable [6]. Whereas Strünker et al. observed that the membrane potential in human sperm was weakly activated by $\mathrm{pH}$ and more strongly by $\mathrm{Ca}^{2+}$, a finding that differed from that observed for the mSlo3 channel [12]. In our study, the SLO3 mutation impaired the membrane potential of mutant sperm after capacitation. In addition, the expression of the SLO3 auxiliary subunit LRRC52 was significantly reduced in sperm from the SLO3 mutant individual compared with that from control, in accordance with the finding that LRRC52 was completely absent from $\mathrm{Slo}^{-/-}$sperm [24]. This result indicated that the expression of LRRC52 is critically dependent on the presence of SLO3 [24-26]. Moreover, the expression of CatSper1 was similar in both control and effected sperm, indicating that $\mathrm{Ca}^{2+}$ channels were not affected by the mutation in $\mathrm{SLO}$. 
A

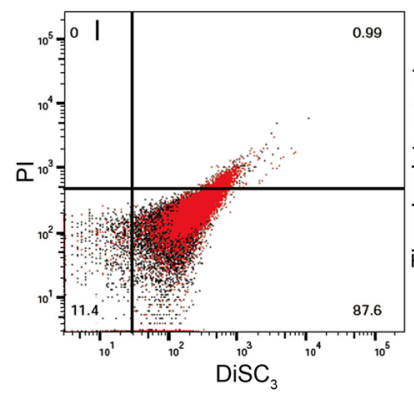

C
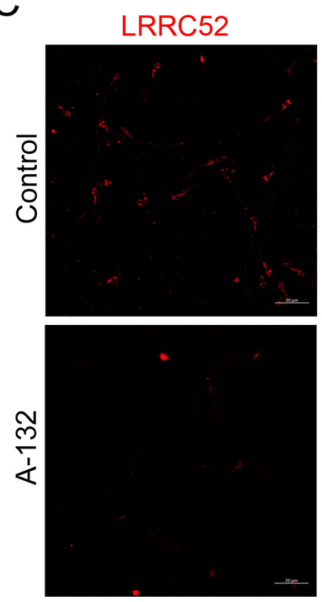

D
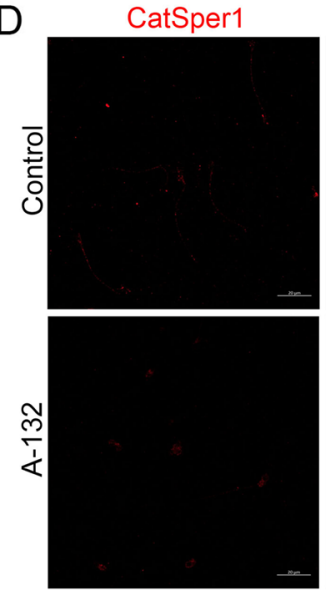

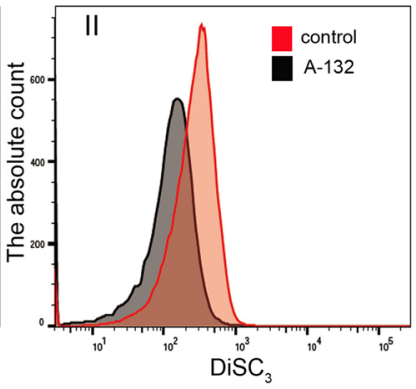

B

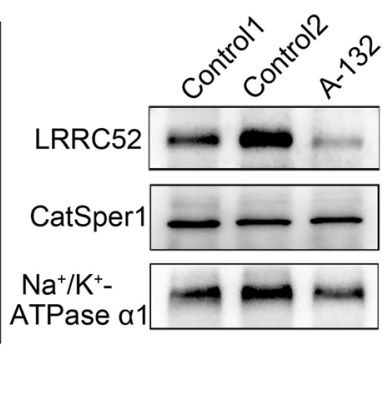

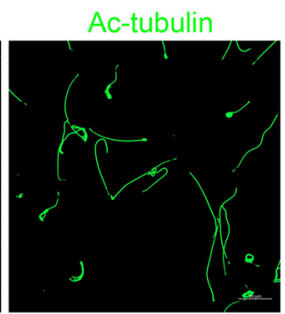
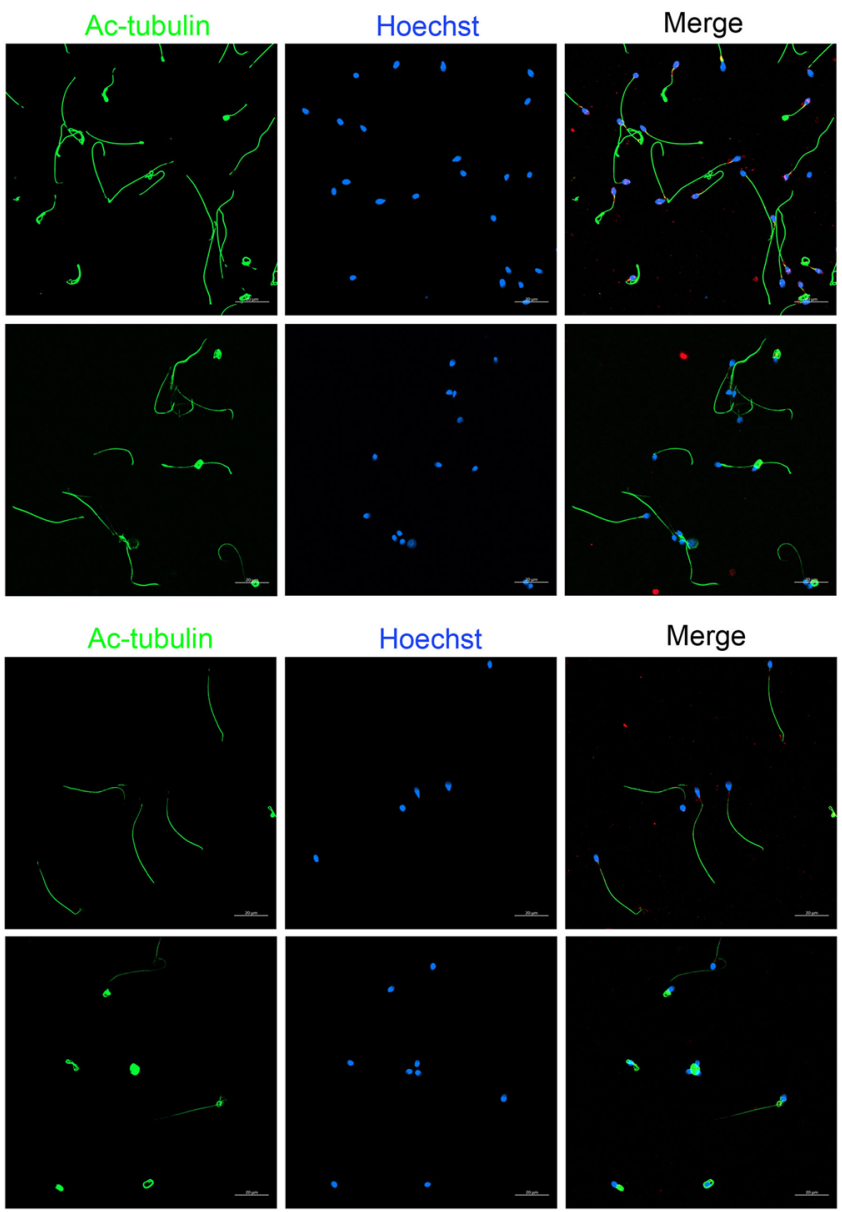

Fig. 6 Membrane potential and expression patterns of LRRC52 and CatSper 1 examined in the spermatozoa from control and SLO3-mutant subjects. A Membrane potential measurements of wild-type and SLO3 mutant sperm in capacitated conditions. The capacitation-associated hyperpolarization of human sperm is inhibited in SLO3 mutant spermatozoa, Data are presented as the mean \pm SEM. ${ }^{* * *} P<0.001$; Student's $t$-test. B Immunoblotting of sperm lysates from controls and the SLO3-mutated individual using the anti-LRRC52 and CatSper1 antibodies. Na ${ }^{+} / \mathrm{K}^{+}-\mathrm{ATPase}$ a1 was used as loading control. The expression of the LRRC52 protein is decreased in mutant sperm, whereas the level of the CatSper 1 protein is comparable to that of control. C Representative images of spermatozoa from fertile controls and the SLO3 mutant patient stained with the anti-LRRC52 antibody, anti-CatSper1 antibody, anti-Ac-tubulin antibody, and Hoechst. Two independent experiments were performed and at least 150 sperm we re-examined for each time per individual. Scale bars, $20 \mu \mathrm{m}$

It has been reported that $S l o 3^{-/-}$male mice were unable to fertilize wild-type females, despite the presence of fertilization plugs [4]. However, partial IVF success was achieved with oocytes harvested from super ovulating wild-type females incubated with capacitated sperm from $\mathrm{Slo3}^{-/-}$male mice [3]. Besides, 
Table 3 Clinical outcomes of ICSI for the SLO3-mutated Man

\begin{tabular}{lll}
\hline & SLO3-mutated Man & OAT as Controls \\
\hline Male age (years) & 29 & $30.1 \pm 4.9$ \\
Female age (years) & 34 & $28.4 \pm 4.2$ \\
No. of ICSI cycles & 1 & 301 \\
No. of oocytes injected & 15 & $11(1,34)$ \\
Fertilization rate (\%) (and number) & $100(15 / 15)$ & $83.3(12.5,100.0)$ \\
& & $(3219 / 4077)$ \\
Cleavage rate (\%) (and number) & $100(15 / 15)$ & $100.0(66.7,100.0)$ \\
8-Cell formation rate (\%) (and number) & $73(11 / 15)$ & $(3188 / 3219)$ \\
Blastocyst formation rate (\%) (and number) & $73(11 / 15)$ & $70.6(10.5,100.0)$ \\
No. of transfer cycles & 1 & $60.0(0,100.0)$ \\
Number of embryos transferred per cycle & 2 & 301 \\
Implantation rate (\%) & 50 & $2(1,2)$ \\
Clinical pregnancy rate (\%) & 100 & $47.9(229 / 478)$ \\
Miscarriage rate (\%) & 0 & $57.1(172 / 301)$ \\
\hline OATOligoast & $11.6(20 / 172)$ \\
\hline
\end{tabular}

OAT Oligoasthenoteratozoospermia; The number of OAT couples is 301

ICSI has become an effective method to help infertile couples, particular for infertile men with asthenoteratozoospermia, achieve a successful pregnancy. In this study, we noticed that the fertilization, cleavage, 8-cell and blastocyst formation rates were similar when performing ICSI with sperm from individual harbouring SLO3 variant and control sperm. We froze 9 blastocysts and 6 months later, transferred 2 blastocysts, resulting in the detection of a single foetal heartbeat via ultrasound; the pregnancy is ongoing. Therefore, our study indicated that male infertility caused by a homozygous SLO3 variant could be rescued by ICSI treatment, which is meaningful for the genetic counselling of patients with SLO3-associated asthenoteratozoospermia.

\section{Conclusion}

In conclusion, our findings on the homozygous mutation of SLO3 might further broaden our knowledge on genetic pathologies associated with male infertility due to severe asthenoteratozoospermia. Our findings might also have important clinical implications for the genetic and reproductive counselling of affected families. Deletion of $\mathrm{SLO} 3$ would seriously impact acrosome formation, mitochondrial sheath assembly, and the function of $\mathrm{K}^{+}$channels. However, there were certain limitations in the present study. Further investigations identifying the functional role of $\mathrm{SLO} 3$ in acrosome formation, mitochondrial sheath assembly are required, and such investigations might involve the screening of a large number of patients with severe asthenoteratozoospermia.

\section{Abbreviations}

WES: Whole-exome sequencing; HE: Haematoxylin and eosin; SEM: Scanning electron microscopy; TEM: Transmission electron microscopy; ICSI: Intra-cytoplasmic sperm injection; qRT-PCR: Quantitative real-time PCR.

\section{Supplementary Information}

The online version contains supplementary material available at https://doi. org/10.1186/s12958-021-00880-4.

Additional file 1: Table S1. Primers Used for Amplification and Verification of SLO3 Mutations. Table S2. Primers Used for qRT-PCR Assays Primer.

\section{Acknowledgments}

We would like to thank the families for participating and supporting this study. We also thank the Center of Cryo-Electron Microscopy at Zhejiang University.

\section{Authors' contributions}

Mingrong Lv, Xiaojin He, Chunyu Liu, Chunjie Ma, Hui Yu and Zhongmei Shao contributed equally to this work. Yunxia Cao, Feng Zhang and Xiaojin He conceived and designed the experiments. Qing Tan, Chuan Xu, Geng Hao, Jingjing Zhang, Hang Li, Xiaohong Mao, Lei Ge, Feifei Fu, Kaixin Zhong collected the clinical samples and analysed sperm. Junqiang Zhang, Yang Gao, Yiyuan Liu, Dongdong Tang and Kuokuo Li organized the medical records and analyzed the whole exome data. Mingrong Lv, Chunyu Liu, Chunjie Ma and Hui Yu performed the experiments and analyzed the experiments data. Yunxia Cao, Feng Zhang, Xiaojin He and Mingrong Lv drafted the article and revised the manuscript. Fangbiao Tao, Yuping Xu, Ping Zhou and Zhaolian Wei provided important guidance for the study. All authors approved the final manuscript to be published and were accounted for all aspects of the work.

\section{Funding}

This work was supported by the National Natural Science Foundation of China (82071705, 81971441 and 31521003), the open project of NHC Key Laboratory of Male Reproduction and Genetics (KF201907), the University Outstanding Young Talents Support Program (gxyq2021174), Major Science and Technology Project of Anhui Province (202003a07020012), and Scientific Research (TP202002) from Anhui Medical University, the University Synergy Innovation Program of Anhui Province (GXXT-2021-071). 


\section{Availability of data and materials}

The datasets used and/or analysed during the current study are available from the corresponding author on reasonable request.

\section{Declarations}

Ethics approval and consent to participate

This study was approved and consented by the Ethics Committee of the First Affiliated Hospital of Anhui Medical University.

\section{Consent for publication}

All the individuals and their family members, as well as the controls signed written informed consents after having received complete information about the research.

\section{Competing interests}

The authors declare no competing interests.

\section{Author details}

${ }^{1}$ Reproductive Medicine Center, Department of Obstetrics and Gynecology, the First Affiliated Hospital of Anhui Medical University, Hefei 230022, Anhui, China. ${ }^{2} \mathrm{NHC}$ Key Laboratory of Study on Abnormal Gametes and Reproductive Tract (Anhui Medical University), Hefei 230032, Anhui, China. ${ }^{3}$ Key Laboratory of Population Health Across Life Cycle, Anhui Medical University, Ministry of Education of the People's Republic of China, Hefei 230032, China. ${ }^{4}$ Obstetrics and Gynecology Hospital, State Key Laboratory of Genetic Engineering at School of Life Sciences, Institute of Reproduction and Development, Fudan University, Shanghai 200011, China. ${ }^{5}$ Shanghai Key Laboratory of Female Reproductive Endocrine Related Diseases, Shanghai 200011, China. ${ }^{6} \mathrm{NHC}$ Key Laboratory of Reproduction Regulation, Shanghai Institute for Biomedical and Pharmaceutical Technologies, Fudan University, Shanghai 200011, China. ${ }^{7} \mathrm{NHC}$ Key Laboratory of Male Reproduction and Genetics, Guangdong Provincial Reproductive Science Institute (Guangdong Provincial Fertility Hospital), Guangzhou 510600, China. ${ }^{8}$ Department of Obstetrics and Gynecology, Fuyang Hospital of Anhui Medical University, Fuyang, China. ${ }^{9}$ Anhui Province Key Laboratory of Reproductive Health and Genetics, Anhui Medical University, Hefei, China. ${ }^{10}$ Anhui Provincial Human Sperm Bank, the First Affiliated Hospital of Anhui Medical University, Hefei, China.

Received: 25 August 2021 Accepted: 10 December 2021 Published online: 03 January 2022

\section{References}

1. Miller D, Vukina J. Recent advances in clinical diagnosis and treatment of male factor infertility. Postgrad Med. 2020;132:28-34.

2. Saberiyan M, Mirfakhraie R, Gholami D, Dehdehi L, Teimori H. Investigating the regulatory function of the ANO1-AS2 on the ANO1 gene in infertile men with asthenozoospermia and terato-asthenozoospermia. Exp Mol Pathol. 2020;117:104528.

3. Santi CM, Martinez-Lopez P, de la Vega-Beltran JL, Butler A, Alisio A, Darszon A, et al. The SLO3 sperm-specific potassium channel plays a vital role in male fertility. FEBS Lett. 2010;584:1041-6.

4. Zeng XH, Yang C, Kim ST, Lingle CJ, Xia XM. Deletion of the Slo3 gene abolishes alkalization-activated $\mathrm{K}+$ current in mouse spermatozoa. Proc Natl Acad Sci U S A. 2011;108:5879-84.

5. Schreiber M, Wei A, Yuan A, Gaut J, Saito M, Salkoff L. Slo3, a novel pHsensitive K+ channel from mammalian spermatocytes. J Biol Chem. 1998;273:3509-16.

6. Leonetti MD, Yuan P, Hsiung Y, Mackinnon R. Functional and structural analysis of the human SLO3 pH- and voltage-gated K+ channel. Proc Natl Acad Sci U S A. 2012;109:19274-9.

7. Zeng XH, Navarro B, Xia XM, Clapham DE, Lingle CJ. Simultaneous knockout of $\mathrm{Slo} 3$ and CatSper1 abolishes all alkalization- and voltage-activated current in mouse spermatozoa. J Gen Physiol. 2013;142:305-13.

8. Geng Y, Ferreira JJ, Dzikunu V, Butler A, Lybaert P, Yuan P, et al. A genetic variant of the sperm-specific SLO3 $\mathrm{K}(+)$ channel has altered $\mathrm{pH}$ and ca (2+) sensitivities. J Biol Chem. 2017;292:8978-87.
9. Santi CM, Butler A, Kuhn J, Wei A, Salkoff L. Bovine and mouse SLO3 K+ channels: evolutionary divergence points to an RCK1 region of critical function. J Biol Chem. 2009;284:21589-98.

10. De La Vega-Beltran JL, Sanchez-Cardenas C, Krapf D, Hernandez-Gonzalez EO, Wertheimer E, Trevino CL, et al. Mouse sperm membrane potential hyperpolarization is necessary and sufficient to prepare sperm for the acrosome reaction. J Biol Chem. 2012;287:44384-93.

11. Demarco IA, Espinosa F, Edwards J, Sosnik J, De La Vega-Beltran JL, Hockensmith JW, et al. Involvement of a $\mathrm{Na}+/ \mathrm{HCO}-3$ cotransporter in mouse sperm capacitation. J Biol Chem. 2003;278:7001-9.

12. Brenker C, Zhou Y, Muller A, Echeverry FA, Trotschel C, Poetsch A, et al. The $\mathrm{Ca} 2+-$ activated $\mathrm{K}+$ current of human sperm is mediated by Slo3. Elife. 2014;3:e01438.

13. Lopez-Gonzalez I, Torres-Rodriguez P, Sanchez-Carranza O, Solis-Lopez A, Santi CM, Darszon A, et al. Membrane hyperpolarization during human sperm capacitation. Mol Hum Reprod. 2014;20:619-29.

14. Cooper TG, Noonan E, von Eckardstein S, Auger J, Baker HW, Behre HM, et al. World Health Organization reference values for human semen characteristics. Hum Reprod Update. 2010;16:231-45.

15. Bjorndahl L, Barratt CL, Mortimer D, Jouannet P.'How to count sperm properly': checklist for acceptability of studies based on human semen analysis. Hum Reprod. 2016;31:227-32.

16. He X, Liu C, Yang X, Lv M, Ni X, Li Q, et al. Bi-allelic loss-of-function variants in CFAP58 cause flagellar Axoneme and mitochondrial sheath defects and Asthenoteratozoospermia in humans and mice. Am J Hum Genet. 2020;107:514-26.

17. Auger J, Jouannet P, Eustache F. Another look at human sperm morphology. Hum Reprod. 2016;31:10-23.

18. Tang S, Wang X, Li W, Yang X, Li Z, Liu W, et al. Biallelic mutations in CFAP43 and CFAP44 cause male infertility with multiple morphological abnormalities of the sperm flagella. Am J Hum Genet. 2017;100:854-64.

19. Liu C, He X, Liu W, Yang S, Wang L, Li W, et al. Bi-allelic mutations in TTC29 cause male subfertility with Asthenoteratospermia in humans and mice. Am J Hum Genet. 2019;105:1168-81.

20. He X, Li W, Wu H, Lv M, Liu W, Liu C, et al. Novel homozygous CFAP69 mutations in humans and mice cause severe asthenoteratospermia with multiple morphological abnormalities of the sperm flagella. J Med Genet. 2019;56:96-103.

21. Gardner DK, Schoolcraft WB. Culture and transfer of human blastocysts. Curr Opin Obstet Gynecol. 1999;11:307-11.

22. Vicens A, Vinuesa P, Arenas M, Trevino CL. Analyzing the functional divergence of Slo1 and Slo3 channel subfamilies. Mol Phylogenet Evol. 2019;133:33-41.

23. Zhang $X$, Zeng $X$, Lingle CJ. Slo3 K+ channels: voltage and pH dependence of macroscopic currents. J Gen Physiol. 2006;128:317-36.

24. Yang C, Zeng XH, Zhou Y, Xia XM, Lingle CJ. LRRC52 (leucine-rich-repeatcontaining protein 52), a testis-specific auxiliary subunit of the alkalization-activated Slo3 channel. Proc Natl Acad Sci U S A. 2011;108:19419-24.

25. Kawai T, Miyata H, Nakanishi H, Sakata S, Morioka S, Sasaki J, et al. Polarized Ptdlns $(4,5)$ P2 distribution mediated by a voltage-sensing phosphatase (VSP) regulates sperm motility. Proc Natl Acad Sci U S A. 2019;116:26020-8.

26. Zeng XH, Yang C, Xia XM, Liu M, Lingle CJ. SLO3 auxiliary subunit LRRC52 controls gating of sperm KSPER currents and is critical for normal fertility. Proc Natl Acad Sci U S A. 2015;112:2599-604.

\section{Publisher's Note}

Springer Nature remains neutral with regard to jurisdictional claims in published maps and institutional affiliations. 